\title{
State of the art and challenges in cave minerals studies
}

\author{
Bogdan P. ONAC ${ }^{1,2^{*}} \&$ Paolo FORTI ${ }^{3}$
}

${ }^{1}$ Department of Geology, University of South Florida, 4202 E. Fowler Ave., Tampa, USA

${ }^{2}$ Department of Geology, "Babeş-Bolyai" University, Kogălniceanu 1, 400084, Cluj / "Emil Racoviță̌" Institute of Speleology, Clinicilor 5, 400006 Cluj-Napoca, Romania

${ }^{3}$ Italian Institute of Speleology, University of Bologna, Via Zamboni 67, 40126, Bologna, Italy

Received January 2011; accepted March 2011

Available online 23 April 2011

DOI: $10.5038 / 1937-8602.56 .1 .4$

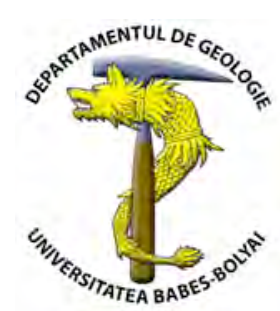

\begin{abstract}
The present note is an updated inventory of all known cave minerals as March 2011. After including the new minerals described since the last edition of the Cave Minerals of the World book (1997) and made the necessary corrections to incorporate all discreditations, redefinitions, or revalidation proposed by the Commission on New Minerals, Nomenclatures and Classification (CNMNC) of the International Mineralogical Association (IMA), we summed up 319 cave minerals, many of these only known from caves. Some of the minerals building up speleothems are powerful tracers of changes in Quaternary climate, other minerals are useful for reconstructing landscape evolution, or allow discriminating between various speleogenetic pathways. Thus, it is expected that the search for new cave minerals will continue and even more attention will be given to those species that carries information that allow for addressing different problems in various earth sciences fields. In view of the exponential increase of cave minerals over the past 50 years, cave mineralogy conceivably has the potential to grow in the future, especially considering the new advances in analytical facilities.
\end{abstract}

Key words: Cave minerals, nomenclature, classification, database.

\section{INTRODUCTION}

Likewise other fields across earth sciences, mineralogy has witnessed over the last decade a rapid growth as a result of fast-paced and revolutionary advances of analytical facilities. For cave mineralogy this translates into an exponential increased of the number of minerals identified (Fig. 1) and described from a variety of cave environments (Onac, 2005, 2011).

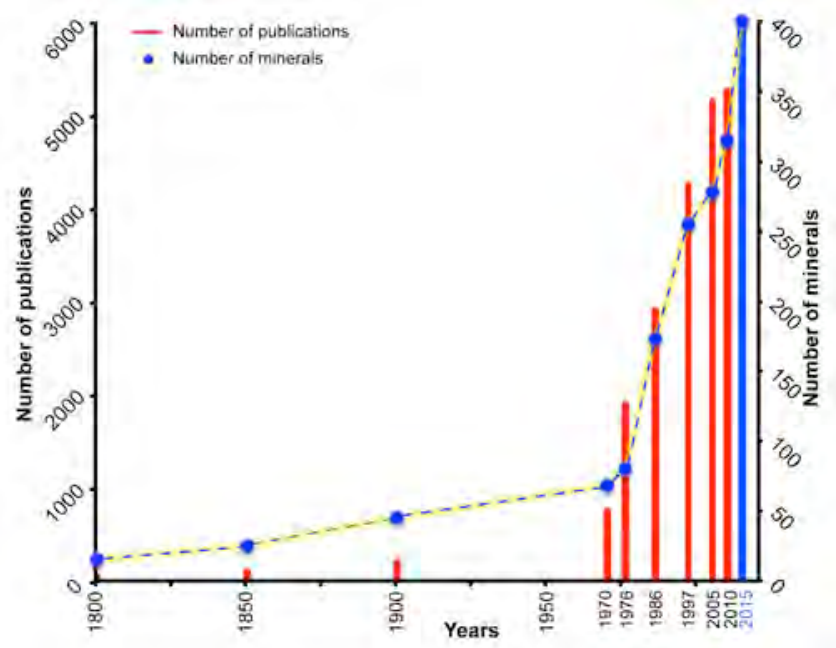

Fig. 1. The evolution of cave mineral studies since 1800; number of minerals vs number of cave minerals-related publications.
Below are some milestones in cave mineral studies since 1750 to present. Although short, non-scientific descriptions on mainly calcite and gypsum from caves are scattered throughout various publications prior to 1750 , the beginning of cave mineralogy as a novel field of research dates back to the second part of the $18^{\text {th }}$ century, when the first detailed presentation of calcite from Dâmbovicioara Cave (Romania) appeared in Mineralogia Magni Principatus Tranylvaniae treatise (Fridvaldszky, 1767). By the beginning of 1800, a total of ten cave minerals were described from Dâmbovicioara, Stufe di San Calogero, Alum, and Pulo di Molfetta caves in Romania and Italy (Forti, 2002; Onac and Forti, 2011). Detailed studies carried out at the end of the $19^{\text {th }}$ century on some cave deposits from Mona Island, USA, and Australia, increased the number of cave minerals and related publications on this topic to about 50 and 250 , respectively. Moore (1970), published a checklist of 68 cave minerals from around the world. The exponential trend seen in Fig. 1 has its origin, however, in the publication of Cave Minerals book (Hill, 1976). Although it includes only 80 minerals, primarily described from United States caves, the number of publications cited nearly topped 2000. Subsequently, Hill and Forti $(1986,1997)$ published two editions of the Cave Minerals of the World pushing the number of cave minerals to 173 and 255, respectively. About half of these minerals (i.e., 86 and 125) were either ore-related or were precipitated in some very particular cave settings (sulfuric-acid caves, lava tubes, caves hosting large quantities of guano, gypsum caves, etc.). It was not only the 
number of cave minerals that increased, but also the number of publications dedicated to them, which exceeded 4000 titles in the $2^{\text {nd }}$ edition of their book. At the threshold of the third millennium, almost 275 minerals had been described in over 5000 publications (Forti, 2002; Hill and Forti, 2007), and these numbers are going up every year (e.g., Onac, 2005, 2011; Forti et al., 2006; Onac et al., 2007a, 2009a, 2011).

\section{CHALLENGES IN CAVE MINERAL STUDIES}

Although it is a major factor in cave mineral investigations, the nature of each sample and the available analytical resources aspect has not been often discussed within the cave science community. Yet the success of a detailed and accurate mineral description depends precisely on which investigation method is used and how well the sample was preserved since the time of collection, until it is ready to be analyzed. As a consequence of the analytical challenges posed, details of various cave mineralizations (e.g., earthy crusts and powders) remain unknown. Even in the case of the growth of calcite or aragonite helictites and eccentrics, with studies spanning several decades focused on these issues - formation mechanisms remain ambiguous.

The main analytical challenges associated to cave mineral investigations are related to (i) the complexity of heterogeneous natural samples, which often contain multiple mineral phases, (ii) extreme sample environments, and (iii) the size and nature of some of the compounds. However, recent developments in a suite of techniques (e.g., X-ray powder or single-crystal diffraction, X-ray fluorescence, inductively coupled plasma-mass spectrometry, electron microprobe, scanning electron microscopy, stable isotopes, etc.) give mineralogists unprecedented opportunities to advance the understanding of caves as minoregenic environments. The results of cave minerals studies, when integrating stable isotope analyses with other microanalytical techniques, can be reassembled to test and improve conceptual ideas in mineral precipitation and to quantify the geochemical processes associated with it.

\section{ABOUT THIS NEW CAVE MINERALS LIST}

Because mineral nomenclature has suffered a number of revisions (Nickel and Grice, 1998; Burke, 2006, 2008;
Mills et al., 2009; Nickel and Nichols, 2009; Pasero et al., 2010) and new cave minerals were reported since 1997 (Rodgers et al., 2000; De Waele and Forti, 2005; Forti, 2005; Forti et al., 2003, 2006, 2007, 2009; Onac \& White, 2003; Onac and Effenberger, 2007; Onac et al., 2002, 2005, 2006, 2009a, b), a compilation of all cave minerals became essential for the cave science community, but not only. The present list incorporates all grandfathered species and species that have been redefined, renamed, or revalidated as proposed by the IMA-CNMNC (Nickel and Nichols, 2009). Also included, but written in italics are names used to designate a group of species (series) and species that were discredited or not approved by the CNMNC. Names of mineral species reported since the last edition of the book Cave Minerals of the World (CMW2; Hill and Forti, 1997) are in bold face.

The format of mineral presentation follows the class scheme presented in the book Dana's New Mineralogy (Gaines et al., 1997), in which each mineral is in alphabetic order, followed but its chemical formula, and the cave type locality of the mineral. The crystal system was included only for polymorphs (e.g., calcite, aragonite, vaterite, etc.) and abbreviations are as follow: monoclinic (mon.), triclinic (tric.), orthorhombic (orth.), tetragonal (tetr.), trigonal (trig.), hexagonal (hex.), and cubic (cub.). To understand the stoichiometry of mineral formulae the charge for altervalent elements is also shown. A vacancy in a structural position is denoted by the $\square$ symbol. With very few exceptions, all mineral formulas included in our compiled list are identical to those reported by Nickel and Nichols (2009). These exceptions were dictated by changes that occurred after the IMA-CNMNC list of minerals was released (e.g., Pasero et al., 2010).

Of the more than 5500 references describing these minerals, over 4000 are listed in Hill and Forti (1997). To keep the length of this note within reasonable limits, we choose to include a selective reference list that contains the major contributions in which the new 63 minerals were reported since 1997. A Commission of Cave Minerals was established in 1997 within the International Union of Speleology with the main purpose of keeping track of all minerals described from caves, including lava tubes and caves intersected by mines.

The official cave mineral database of this commission it is assembled now (work is in progress) and can be accessed at https://www.lib.usf.edu/caveminerals/ca/.

\section{Native elements}

Bismuth - Bi; Oilloki Mine Cave, France (Audra, 2007)

Sulfur - S; Turia Cave, Romania

Gold - Au; Valea Rea Cave, Romania (Ghergari et al., 1997)

\section{Sulfides}

Chalcocite $-\mathrm{Cu}_{2} \mathrm{~S}$; Tyuya-Muyun, Kyrgyzstan

Chalcopyrite $-\mathrm{CuFeS}_{2}$; Magian \& Marguzor caves, Tajikistan

Cinnabar - HgS (trig.); Magian \& Marguzor caves, Tajikistan

Galena - PbS; Dalnegorskaya Cave, Russia

Marcasite - $\mathrm{FeS}_{2}$ (orth.) - Dachstein-Mammuthöhle, Austria

Metacinnabar - HgS (cub.); Fata Morgana caves, Turkmenistan

Orpiment $-\mathrm{As}_{2} \mathrm{~S}_{3}$; Aghia Paraskevi caves, Greece (Lazarides et al., 2011)

Pyrite $-\mathrm{FeS}_{2}$ (cub.); Lauback Cave, TX, USA

Pyrrhotite $-\mathrm{Fe}_{1-\mathrm{x}} \mathrm{S}(\mathrm{x}<0.17)$ (mon.); Dalnegorskaya Cave, Russia 
Realgar - AsS; Chauvai, Kyrgyzstan

Sphalerite - ZnS (cub.); Trzebionka mine caves, Poland

Stibnite $-\mathrm{Sb}_{2} \mathrm{~S}_{3}$; Alay Ridge caves, Kyrgyzstan

\section{Oxides \& Hydroxides}

Akaganeite - $\left(\mathrm{Fe}^{3+}, \mathrm{Ni}^{2+}\right)_{8}(\mathrm{OH}, \mathrm{O})_{16} \mathrm{Cl}_{1.25} \cdot n \mathrm{H}_{2} \mathrm{O}$; Ruatapu Cave, New Zealand (Rodgers et al., 2000)

Arsenolite - $\mathrm{As}_{2} \mathrm{O}_{3}$ (cub.); Corkscrew Cave, AZ, USA (Onac et al., 2007b)

Asbolane $-\mathrm{Mn}^{4+}(\mathrm{O}, \mathrm{OH})_{2}(\mathrm{Co}, \mathrm{Ni}, \mathrm{Mg}, \mathrm{Ca})_{\mathrm{x}}(\mathrm{OH})_{2 \times} \cdot n \mathrm{H}_{2} \mathrm{O}$; Tyuya-Muyun, Kyrgyzstan

Birnessite $-(\mathrm{Na}, \mathrm{Ca}, \mathrm{K})_{0.6}\left(\mathrm{Mn}^{4+}, \mathrm{Mn}^{3+}\right)_{2} \mathrm{O}_{4} \cdot 1.5 \mathrm{H}_{2} \mathrm{O}$; Weber Cave, IA, USA

Böhmite - $\mathrm{AlO}(\mathrm{OH})$ (orth.); Dachstein-Mammuthöhle, Austria

Braunite - $\mathrm{Mn}^{2+}\left(\mathrm{Mn}^{3+}\right)_{6} \mathrm{SiO}_{12}$; Vântului Cave, Romania

Cesàrolite - $\mathrm{Pb}\left(\mathrm{Mn}^{4+}\right)_{3} \mathrm{O}_{6}(\mathrm{OH})_{2}$; in Santa Barbara Cave, Sardinia, Italy (De Waele and Forti, 2005)

Chalcophanite $-\mathrm{Zn}\left(\mathrm{Mn}^{4+}\right)_{3} \mathrm{O}_{7} \cdot 3 \mathrm{H}_{2} \mathrm{O}$; Bisbee mine cave, AZ, USA

Claudetite $-\mathrm{As}_{2} \mathrm{O}_{3}$ (mon.); Corkscrew Cave, AZ, USA (Onac et al., 2007b)

Coronadite $-\mathrm{Pb}\left(\mathrm{Mn}^{4+}\right)_{2}\left(\mathrm{Mn}^{2+}\right)_{6} \mathrm{O}_{16}$; in Santa Barbara Cave, Sardinia, Italy (?) (De Waele and Forti, 2005)

Cristobalite $-\mathrm{SiO}_{2}$ (tetr.); Medicine Lake lava tubes, California, USA

Cryptomelane $-\mathrm{K}\left(\mathrm{Mn}^{4+}, \mathrm{Mn}^{2+}\right)_{8} \mathrm{O}_{16}$; cavities in Nikopol'skoye iron ore, Russia

Cuprite $-\mathrm{Cu}_{2} \mathrm{O}$; Bisbee mine cave, AZ, USA

Gibbsite $-\mathrm{Al}(\mathrm{OH})_{3}$ (mon.); Harlansburg \& Hineman caves, PA, USA

Goethite - $\mathrm{FeO}(\mathrm{OH})$ (orth.); Tintic district, UT, USA

Hausmannite $-\mathrm{Mn}^{2+}\left(\mathrm{Mn}^{3+}\right)_{2} \mathrm{O}_{4}$; Vântului Cave, Romania

Hematite $-\mathrm{Fe}_{2} \mathrm{O}_{3}$; cavities in the Tintic district, UT, USA

Hetaerolite $-\mathrm{Zn}\left(\mathrm{Mn}^{3+}\right)_{2} \mathrm{O}_{4}$; in Santa Barbara Cave, Sardinia, Italy (De Waele and Forti, 2005)

Hollandite - $(\mathrm{Ba}, \mathrm{K}, \mathrm{Ca}, \mathrm{Sr})\left(\mathrm{Mn}^{4+}, \mathrm{Mn}^{3+}, \mathrm{Ti}_{1} \mathrm{Fe}^{3+}\right)_{8} \mathrm{O}_{16}$; Vântului Cave, Romania (Onac et al., 1997)

Hydrohetaerolite - $\mathrm{HZn}\left(\mathrm{Mn}^{3+}\right)_{1.7} \mathrm{O}_{4}$; in Santa Barbara Cave, Sardinia, Italy (De Waele and Forti, 2005)

Ice $-\mathrm{H}_{2} \mathrm{O}$; Kungur Cave, Russia

Lepidocrocite $-\mathrm{Fe}^{3+} \mathrm{O}(\mathrm{OH})$ (orth.); Dachstein-Mammuthöhle, Austria

Limonite - NOT a mineral but generic term used for undifferentiated hydrated iron oxides; NOT approved by IMA-CNMNC

Lithiophorite - $(\mathrm{Al}, \mathrm{Li}) \mathrm{Mn}^{4+} \mathrm{O}_{2}(\mathrm{OH})_{2}$; Martel Cave, Venezuela

Maghemite - $(\mathrm{Fe}, \sim)_{3} \mathrm{O}_{4}$; Cueva la Milagrosa, Venezuela

Magnetite $-\mathrm{Fe}^{2+}\left(\mathrm{Fe}^{3+}\right)_{2} \mathrm{O}_{4}$; Dachstein-Mammuthöhle, Austria

Manganite $-\mathrm{Mn}^{3+} \mathrm{O}(\mathrm{OH})$ (mon.); Najdema Cave, Slovenia

Monteponite - CdO; cave in Monteponi mine, Sardinia

Nordstrandite $-\mathrm{Al}(\mathrm{OH})_{3}$ (tric.); Lechuguilla Cave, USA (Polyak and Provencio, 2001)

Opal $-\mathrm{SiO}_{2} \cdot n \mathrm{H}_{2} \mathrm{O}$; Doombera granite cave, Ceylon

Periclase - $\mathrm{MgO}$; Valea Rea Cave, Romania

Plattnerite $-\mathrm{PbO}_{2}$; Bisbee mine cave, AZ, USA

Portlandite $-\mathrm{Ca}(\mathrm{OH})_{2}$; lava tube on Mt. Etna, Italy

Pyrolusite $-\mathrm{MnO}_{2}$; Blue John Cavern, UK

Quartz - $\mathrm{SiO}_{2}$ (trig.); granite cave near Rio de Janiero, Brazil

Ralstonite $-\mathrm{Na}_{0.5}(\mathrm{Al}, \mathrm{Mg})_{2}(\mathrm{~F}, \mathrm{OH})_{6} \cdot \mathrm{H}_{2} \mathrm{O}$; volcanic caves on Surtsey Island, Iceland

Ranciéite - $\left(\mathrm{Ca}, \mathrm{Mn}^{2+}\right)_{0.2}\left(\mathrm{Mn}^{4+}, \mathrm{Mn}^{3+}\right) \mathrm{O}_{2} \cdot 0.6 \mathrm{H}_{2} \mathrm{O}$; cave type locality unknown

Romanèchite - $\left(\mathrm{Ba}, \mathrm{H}_{2} \mathrm{O}\right)_{2}\left(\mathrm{Mn}^{4+}, \mathrm{Mn}^{3+}\right)_{5} \mathrm{O}_{10}$; Zbrasov Cave, Czech Republic

Silhydrite $-\mathrm{Si}_{3} \mathrm{O}_{6} \cdot \mathrm{H}_{2} \mathrm{O}$; Gaping Hole/Arch Sink, Mammoth, and Table Mountain, lava tubes in California, USA

Tenorite - $\mathrm{CuO}$; Bisbee mine cave, AZ, USA

Todorokite $-(\mathrm{Na}, \mathrm{Ca}, \mathrm{K}, \mathrm{Ba}, \mathrm{Sr})_{1-\mathrm{x}}(\mathrm{Mn}, \mathrm{Mg}, \mathrm{Al})_{6} \mathrm{O}_{12} \cdot 3-4 \mathrm{H}_{2} \mathrm{O}$; caves in $\mathrm{KY}, \mathrm{TN}, \mathrm{GA}$ (USA)

Tridymite $-\mathrm{SiO}_{2}$ (tric.); Cango Cave, South Africa

Vernadite $-(\mathrm{Mn}, \mathrm{Fe}, \mathrm{Ca}, \mathrm{Na})(\mathrm{O}, \mathrm{OH})_{2} \cdot n \mathrm{H}_{2} \mathrm{O}$; Shopov's cave system, Bulgaria (?)

Wad - NOT a mineral but a generic term used for poorly crystalline, undifferentiated hydrated manganese oxides and hydroxides; NOT approved by IMA-CNMNC

Woodruffite $-\mathrm{Zn}_{2}\left(\mathrm{Mn}^{4+}\right)_{5} \mathrm{O}_{12} \cdot 4 \mathrm{H}_{2} \mathrm{O}$; Cueva de las Velas, Naica, Mexico (Forti et al., 2007)

\section{Halogenides}

Atacamite $-\mathrm{Cu}_{2} \mathrm{Cl}(\mathrm{OH})_{3}$ (orth.); Jingemia Cave, Australia

Bromargyrite - AgBr; Bisbee mine cave, AZ, USA

Carnallite $-\mathrm{KMgCl}_{3} \cdot 6 \mathrm{H}_{2} \mathrm{O}$; Verhnekamsk caves, Russia

Chlormagnesite $-\mathrm{MgCl}_{2}$; Pelagalli Cave, Italy

Fluorite $-\mathrm{CaF}_{2}$; Blue John Cavern, UK

Halite - NaCl; cave type locality unknown

Salammoniac $-\mathrm{NH}_{4} \mathrm{Cl}$; volcanic cave on Vulcano Island, Italy; Renamed with approval from IMA-CNMNC

Sylvite - KCl; Cutrona Cave Mt. Etna, Italy 


\section{Carbonates}

Aragonite - $\mathrm{CaCO}_{3}$ (orth.); cave type locality unknown

Ankerite $-\mathrm{CaFe}^{2+}\left(\mathrm{CO}_{3}\right)_{2}$; cave in Russia

Artinite $-\mathrm{Mg}_{2} \mathrm{CO}_{3}(\mathrm{OH})_{2} \cdot 3 \mathrm{H}_{2} \mathrm{O}$; Shopov's cave system, Bulgaria

Aurichalcite $-\mathrm{Zn}_{5}\left(\mathrm{CO}_{3}\right)_{2}(\mathrm{OH})_{6}$; Blanchard mine caves, NM, USA

Azurite - $\mathrm{Cu}_{3}\left(\mathrm{CO}_{3}\right)_{2}(\mathrm{OH})_{2}$; Bisbee mine cave, AZ, USA

Baylissite $-\mathrm{K}_{2} \mathrm{Mg}\left(\mathrm{CO}_{3}\right)_{2} \cdot 4 \mathrm{H}_{2} \mathrm{O}$; Shopov's cave system, Bulgaria

Brianyoungite $-\mathrm{Zn}_{3} \mathrm{CO}_{3}(\mathrm{OH})_{4}$; cave in Su Zurfuru mine, Sardinia, Italy (De Waele and Forti, 2005)

Burbankite - $(\mathrm{Na}, \mathrm{Ca})_{3}(\mathrm{Sr}, \mathrm{Ba}, \mathrm{Ce})_{3}\left(\mathrm{CO}_{3}\right)_{5}$; Cioclovina Cave, Romania (Onac et al., 2009a)

Calcite $-\mathrm{CaCO}_{3}$ (trig.); cave in the Julian Alps, Italy

Cerussite $-\mathrm{PbCO}_{3}$; Blue John Cavern, UK (?)

Dolomite - $\mathrm{CaMg}\left(\mathrm{CO}_{3}\right)_{2}$; cave in France

Gaspéite - $\mathrm{NiCO}_{3}$; cave in San Benedetto mine, Sardinia, Italy (De Waele and Forti, 2005)

Glaukosphaerite - $(\mathrm{Cu}, \mathrm{Ni})_{2} \mathrm{CO}_{3}(\mathrm{OH})_{2}$; Water Cave from Codreanu mine, Romania (Onac, 2002)

Huntite $-\mathrm{CaMg}_{3}\left(\mathrm{CO}_{3}\right)_{4}$; Dorog, Hungary

Hydromagnesite $-\mathrm{Mg}_{5}\left(\mathrm{CO}_{3}\right)_{4}(\mathrm{OH})_{2} \cdot 4 \mathrm{H}_{2} \mathrm{O}$; Carlsbad Caverns, NM, USA

Hydrozincite $-\mathrm{Zn}_{5}\left(\mathrm{CO}_{3}\right)_{2}(\mathrm{OH})_{6}$; Island Ford Cave, VA, USA

Ikaite $-\mathrm{CaCO}_{3} \cdot 6 \mathrm{H}_{2} \mathrm{O}$; Scărişoara Cave, Romania (Onac, 2008)

Kutnohorite - $\mathrm{CaMn}^{2+}\left(\mathrm{CO}_{3}\right)_{2}$; Caverna Pocala, Italy

Lansfordite $-\mathrm{MgCO}_{3} \cdot 5 \mathrm{H}_{2} \mathrm{O}$; Valea Rea Cave, Romania (Onac, 2003)

Magnesite $-\mathrm{MgCO}_{3}$; Moulis Cave, France (?)

Malachite $-\mathrm{Cu}_{2} \mathrm{CO}_{3}(\mathrm{OH})_{2}$; Bisbee mine cave, AZ, USA

Monohydrocalcite $-\mathrm{CaCO}_{3} \cdot \mathrm{H}_{2} \mathrm{O}$; Eibengrotte, Germany

Natron $-\mathrm{Na}_{2} \mathrm{CO}_{3} \cdot 10 \mathrm{H}_{2} \mathrm{O}$; Pisgah lava tubes, CA, USA

Nesquehonite $-\mathrm{MgCO}_{3} \cdot 3 \mathrm{H}_{2} \mathrm{O}$; Wolkberg Cave, South Africa

Norsethite - $\mathrm{BaMg}\left(\mathrm{CO}_{3}\right)_{2}$; Crystal's Cave, Codreanu mine, Romania (Onac, 2002)

Paralstonite - $\mathrm{BaCa}\left(\mathrm{CO}_{3}\right)_{2}$; Cova des Pas de Vallgornera, Mallorca, Spain (Merino et al., 2009)

Phosgenite $-\mathrm{Pb}_{2} \mathrm{CO}_{3} \mathrm{Cl}_{2}$; cave in Monteponi mine, Sardinia, Italy (De Waele and Forti, 2005)

Rhodochrosite $-\mathrm{MnCO}_{3}$; cavities in Andalgala mining district, Argentina

Rosasite - $(\mathrm{Cu}, \mathrm{Zn})_{2} \mathrm{CO}_{3}(\mathrm{OH})_{2}$; Bisbee mine cave, AZ, USA

Siderite $-\mathrm{FeCO}_{3}$; Jewel Cave, SD, USA

Smithsonite $-\mathrm{ZnCO}_{3}$; cave in Iowa, USA

Strontianite - $\mathrm{SrCO}_{3}$; cave in Terrace Mountain, NY, USA

Thermonatrite $-\mathrm{Na}_{2} \mathrm{CO}_{3} \cdot \mathrm{H}_{2} \mathrm{O}$; Salzburgerschacht, Austria

Trona - $\mathrm{Na}_{3}\left(\mathrm{HCO}_{3}\right)\left(\mathrm{CO}_{3}\right) \cdot 2 \mathrm{H}_{2} \mathrm{O}$; Pisgah lava tubes, $\mathrm{CA}$, USA

Vaterite $-\mathrm{CaCO}_{3}$ (hex.); Closani Cave, Romania

Witherite $-\mathrm{BaCO}_{3}$; Lilburn Cave, CA, USA

\section{Nitrates}

Darapskite - $\mathrm{Na}_{3}\left(\mathrm{SO}_{4}\right)\left(\mathrm{NO}_{3}\right) \cdot \mathrm{H}_{2} \mathrm{O}$; Flower Cave, TX, USA

Gwihabaite - $\left(\mathrm{NH}_{4}\right) \mathrm{NO}_{3}$; Gwihaiba Cave, Botswana

Hydrombobomkulite - $(\mathrm{Ni}, \mathrm{Cu}) \mathrm{Al}_{4}\left(\mathrm{NO}_{3}\right)_{2}\left(\mathrm{SO}_{4}\right)(\mathrm{OH})_{12} \cdot 14 \mathrm{H}_{2} \mathrm{O}$; Mbobo Mkulu Cave, South Africa

Mbobomkulite - $(\mathrm{Ni}, \mathrm{Cu}) \mathrm{Al}_{4}\left(\mathrm{NO}_{3}, \mathrm{SO}_{4}\right)_{2}(\mathrm{OH})_{12} \cdot 3 \mathrm{H}_{2} \mathrm{O}$; Mbobo Mkulu Cave, South Africa

"Nickelalumite" - not approved by IMA-CNMNC (use mbobomkulite)

Niter $-\mathrm{KNO}_{3}$; Pulo din Molfetta caves, Italy

Nitrammite - $\mathrm{NH}_{4} \mathrm{NO}_{3}$; discredited by IMA-CNMNC (use gwihabaite)

Soda niter - $\mathrm{NaNO}_{3}$; cave type locality unknown

Nitrocalcite $-\mathrm{Ca}\left(\mathrm{NO}_{3}\right)_{2} \cdot 4 \mathrm{H}_{2} \mathrm{O}$; Pulo di Molfetta caves, Italy

Nitromagnesite $-\mathrm{Mg}\left(\mathrm{NO}_{3}\right)_{2} \cdot 6 \mathrm{H}_{2} \mathrm{O}$; Great Cave, KY, USA (?)

Sveite $-\mathrm{KAl}_{7}\left(\mathrm{NO}_{3}\right)_{4}(\mathrm{OH})_{16} \mathrm{Cl}_{2} \cdot 8 \mathrm{H}_{2} \mathrm{O}$; Autana Cave, Venezuela

\section{Borates}

Tincalconite $-\mathrm{Na}_{2} \mathrm{~B}_{4} \mathrm{O}_{5}(\mathrm{OH})_{4} \cdot 3 \mathrm{H}_{2} \mathrm{O}$; Tincalconite Cave, CA, USA

\section{Sulfates}

Alpersite - $\left(\mathrm{Mg}_{,} \mathrm{Cu}^{2+}\right) \mathrm{SO}_{4} \cdot 7 \mathrm{H}_{2} \mathrm{O}$; Cave at $-150 \mathrm{~m}$ in Naica Mine, Mexico (originally described as Cu-pentahydrite; Forti, 2010) Alum- $(\mathrm{K})-\mathrm{KAl}\left(\mathrm{SO}_{4}\right)_{2} \cdot 12 \mathrm{H}_{2} \mathrm{O}$; Doombera granite cave, Ceylon; Renamed with approval from IMA-CNMNC

Alum-(Na) - $\mathrm{NaAl}\left(\mathrm{SO}_{4}\right)_{2} \cdot 12 \mathrm{H}_{2} \mathrm{O}$; Alum Cave, Italy; Renamed with approval from IMA-CNMNC

Aluminite $-\mathrm{Al}_{2} \mathrm{SO}_{4}(\mathrm{OH})_{4} \cdot 7 \mathrm{H}_{2} \mathrm{O}$; Mbobo Mkulu Cave, South Africa

Aluminocopiapite - $(\mathrm{Al}, \mathrm{Mg})\left(\mathrm{Fe}^{3+}\right)_{4}\left(\mathrm{SO}_{4}\right)_{6}(\mathrm{OH}, \mathrm{O})_{2} \cdot 20 \mathrm{H}_{2} \mathrm{O}$; Alum Cave, Italy

Alunite - $\mathrm{KAl}_{3}\left(\mathrm{SO}_{4}\right)_{2}(\mathrm{OH})_{6}$; Fourgassié Cave, French Guiana

Alunogen - $\mathrm{Al}_{2}\left(\mathrm{SO}_{4}\right)_{3}\left(\mathrm{H}_{2} \mathrm{O}\right)_{12} \cdot 5 \mathrm{H}_{2} \mathrm{O}$; Grotta dello Zolfo, Italy

Ammoniojarosite - $\mathrm{NH}_{4}\left(\mathrm{Fe}^{3+}\right)_{3}\left(\mathrm{SO}_{4}\right)_{2}(\mathrm{OH})_{6}$; Cueva Alfredo Jahn, Venezuela

Studia UBB Geologia, 2011, 56 (1), 33 - 42 
Anglesite - $\mathrm{PbSO}_{4}$; Ahumada mine cave, Mexico

Anhydrite - $\mathrm{CaSO}_{4}$; Diana Cave, Romania

Antlerite - $\left(\mathrm{Cu}^{2+}\right)_{3} \mathrm{SO}_{4}(\mathrm{OH})_{4}$; Cave at $-150 \mathrm{~m}$ in Naica Mine, Mexico (Forti, 2010)

Aphthitalite $-\mathrm{K}_{3} \mathrm{Na}\left(\mathrm{SO}_{4}\right)_{2}$; Murra-el-elevyn Cave, Australia

Arcanite $-\mathrm{K}_{2} \mathrm{SO}_{4}$; Murra-el-elevyn Cave, Australia (originally described as taylorite, name that is now discredited by IMA-CNMNC)

Aubertite $-\mathrm{Cu}^{2+} \mathrm{Al}\left(\mathrm{SO}_{4}\right)_{2} \mathrm{Cl} \cdot 14 \mathrm{H}_{2} \mathrm{O}$; Alum Cave, Italy

Barite - $\mathrm{BaSO}_{4}$; paleokarst cavities in Derbyshire, UK

Basaluminite - Discredited by IMA-CNMNC (use felsöbányaite)

Bassanite $-\mathrm{CaSO}_{4} \cdot 0.5 \mathrm{H}_{2} \mathrm{O}$; Tăuşoare Cave, Romania

Bechererite $-\mathrm{Zn}_{7} \mathrm{Cu}(\mathrm{OH})_{13}\left[\mathrm{SiO}(\mathrm{OH})_{3} \mathrm{SO}_{4}\right]$; cave in Su Zurfuru mine, Sardinia, Italy (De Waele and Forti, 2005)

Bianchite $-\mathrm{ZnSO}_{4} \cdot 6 \mathrm{H}_{2} \mathrm{O}$; cave in Campo Pisano mine, Sardinia, Italy (De Waele and Forti, 2005)

Blödite $-\mathrm{Na}_{2} \mathrm{Mg}\left(\mathrm{SO}_{4}\right)_{2} \cdot 4 \mathrm{H}_{2} \mathrm{O}$; Lee Cave (Mammoth cave system), USA

Boussingaultite $-\left(\mathrm{NH}_{4}\right)_{2} \mathrm{Mg}\left(\mathrm{SO}_{4}\right)_{2} \cdot 6 \mathrm{H}_{2} \mathrm{O}$; Gwihaiba Cave, Botswana

Brochantite $-\mathrm{Cu}_{4} \mathrm{SO}_{4}(\mathrm{OH})_{6}$; Bingham mine caves, USA

Burkeite - $\mathrm{Na}_{4}\left(\mathrm{SO}_{4}\right)\left(\mathrm{CO}_{3}\right)$; El Malpais lava tubes, USA

Celestine - $\mathrm{SrSO}_{4}$; Crystal Cave, Ohio, USA

Cesanite $-\mathrm{Na}_{7} \mathrm{Ca}_{3}\left(\mathrm{SO}_{4}\right)_{6}(\mathrm{OH}) \cdot \mathrm{H}_{2} \mathrm{O}$; Lighthouse Cave, San Salvador, Bahamas (Onac et al., 2001a)

Chalcanthite $-\mathrm{CuSO}_{4} \cdot 5 \mathrm{H}_{2} \mathrm{O}$; Bisbee mine caves, USA

Chalcoalumite $-\mathrm{CuAl}_{4} \mathrm{SO}_{4}(\mathrm{OH})_{12} \cdot 3 \mathrm{H}_{2} \mathrm{O}$; Mbobo Mkulu Cave, South Africa

Clairite - $\left(\mathrm{NH}_{4}\right)_{2}\left(\mathrm{Fe}^{3+}\right)_{3}\left(\mathrm{SO}_{4}\right)_{4}(\mathrm{OH})_{3} \cdot 3 \mathrm{H}_{2} \mathrm{O}$; Lone Creek Fall Cave, South Africa

Copiapite $-\mathrm{Fe}^{2+}\left(\mathrm{Fe}^{3+}\right)_{4}\left(\mathrm{SO}_{4}\right)_{6}(\mathrm{OH})_{2} \cdot 20 \mathrm{H}_{2} \mathrm{O}$; Alum Cave, Italy

Coquimbite $-\left(\mathrm{Fe}^{3+}\right)_{2}\left(\mathrm{SO}_{4}\right)_{3} \cdot 9 \mathrm{H}_{2} \mathrm{O} ;$ Alum Cave, Italy

Cyanotrichite $-\mathrm{Cu}_{4} \mathrm{Al}_{2} \mathrm{SO}_{4}(\mathrm{OH})_{12} \cdot 2 \mathrm{H}_{2} \mathrm{O}$; Bingham mine caves, USA

Despujolsite $-\mathrm{Ca}_{3} \mathrm{Mn}^{4+}\left(\mathrm{SO}_{4}\right)_{2}(\mathrm{OH})_{6} \cdot 3 \mathrm{H}_{2} \mathrm{O}$; Shopov's cave system, Bulgaria

Devilline $-\mathrm{CaCu}_{4}\left(\mathrm{SO}_{4}\right)_{2}(\mathrm{OH})_{6} \cdot 3 \mathrm{H}_{2} \mathrm{O}$; Monte Rosso Cave, Italy

Epsomite $-\mathrm{MgSO}_{4} \cdot 7 \mathrm{H}_{2} \mathrm{O}$; gypusm cave near Bologna, Italy

Felsőbányaite - $\mathrm{Al}_{4}\left(\mathrm{SO}_{4}\right)(\mathrm{OH})_{10} \cdot 4 \mathrm{H}_{2} \mathrm{O}$; Tateishi-Shônyû-dô Cave, Japan

Ferrohexahydrite $-\mathrm{Fe}^{2+} \mathrm{SO}_{4} \cdot 6 \mathrm{H}_{2} \mathrm{O}$; Cupp-Coutunn Cave, Turkmenistan

Fibroferrite $-\mathrm{Fe}^{3+} \mathrm{SO}_{4}(\mathrm{OH}) \cdot 5 \mathrm{H}_{2} \mathrm{O}$; Ferrata Cave, Umbria, Italy

Galeite $-\mathrm{Na}_{15}\left(\mathrm{SO}_{4}\right)_{5} \mathrm{ClF}_{4}$; volcanic caves on Surtsey Island, Iceland

Glauberite $-\mathrm{Na}_{2} \mathrm{Ca}\left(\mathrm{SO}_{4}\right)_{2}$; Grillid volcanic cave, Surtsey Island, Iceland

Gypsum - $\mathrm{CaSO}_{4} \cdot 2 \mathrm{H}_{2} \mathrm{O}$; Mammoth cave system, USA

Halotrichite $-\mathrm{Fe}^{2+} \mathrm{Al}_{2}\left(\mathrm{SO}_{4}\right)_{4} \cdot 22 \mathrm{H}_{2} \mathrm{O}$; Alum Cave, Italy

Hexahydrite $-\mathrm{MgSO}_{4} \cdot 6 \mathrm{H}_{2} \mathrm{O}$; Wyandotte Cave, Indiana, USA

Hydrobasaluminite $-\mathrm{Al}_{4} \mathrm{SO}_{4}(\mathrm{OH})_{10} \cdot 15 \mathrm{H}_{2} \mathrm{O}$; Alum Cave, Italy

Hydroglauberite $-\mathrm{Na}_{10} \mathrm{Ca}_{3}\left(\mathrm{SO}_{4}\right)_{8} \cdot 6 \mathrm{H}_{2} \mathrm{O}$; Grillid volcanic cave, Surtsey Island, Iceland

Hydroniumjarosite $-\left(\mathrm{H}_{3} \mathrm{O}\right)\left(\mathrm{Fe}^{3+}\right)_{3}\left(\mathrm{SO}_{4}\right)_{2}(\mathrm{OH})_{6}$; Iza Cave, Romania (?) (Tămaş and Ghergari, 2003)

Jarosite $-\mathrm{K}\left(\mathrm{Fe}^{3+}\right)_{3}\left(\mathrm{SO}_{4}\right)_{2}(\mathrm{OH})_{6}$; Turia Cave, Romania

Jurbanite $-\mathrm{AlSO}_{4}(\mathrm{OH}) \cdot 5 \mathrm{H}_{2} \mathrm{O}$; Serpents Cave, France (Audra and Hobléa, 2007)

Kainite $-\mathrm{KMg}\left(\mathrm{SO}_{4}\right) \mathrm{Cl} \cdot 3 \mathrm{H}_{2} \mathrm{O}$; Grillid volcanic cave, Surtsey Island, Iceland (Forti, 2005)

Kalinite - $\mathrm{KAl}\left(\mathrm{SO}_{4}\right)_{2} \cdot 11 \mathrm{H}_{2} \mathrm{O}$; Alum Cave, Italy

Kieserite $-\mathrm{MgSO}_{4} \cdot \mathrm{H}_{2} \mathrm{O}$; Tana di Val Serrata Cave, Italy

Kogarkoite - $\mathrm{Na}_{3} \mathrm{SO}_{4} \mathrm{~F}$; Cave \#13 (lava tube), Mt. Suswa, Kenya (Forti et al., 2003)

Koktaite - $\left(\mathrm{NH}_{4}\right)_{2} \mathrm{Ca}\left(\mathrm{SO}_{4}\right)_{2} \cdot \mathrm{H}_{2} \mathrm{O}$; Cueva Alfredo Jahn, Venezuela

Konyaite $-\mathrm{Na}_{2} \mathrm{Mg}\left(\mathrm{SO}_{4}\right)_{2} \cdot 5 \mathrm{H}_{2} \mathrm{O}$; Tăuşoare Cave, Romania (Onac et al., 2001b)

Kröhnkite $-\mathrm{Na}_{2} \mathrm{Cu}\left(\mathrm{SO}_{4}\right)_{2} \cdot 2 \mathrm{H}_{2} \mathrm{O}$; Cioclovina Cave, Romania (Onac et al., 2011)

Lecontite $-\left(\mathrm{NH}_{4}\right) \mathrm{Na}\left(\mathrm{SO}_{4}\right) \cdot 2 \mathrm{H}_{2} \mathrm{O}$; cave near Las Piedras, Honduras

Leonite $-\mathrm{K}_{2} \mathrm{Mg}\left(\mathrm{SO}_{4}\right)_{2} \cdot 4 \mathrm{H}_{2} \mathrm{O}$; Tăuşoare Cave, Romania (Onac et al., 2001b)

Lonecreekite $-\mathrm{NH}_{4}\left(\mathrm{Fe}^{3+}\right)\left(\mathrm{SO}_{4}\right)_{2} \cdot 12 \mathrm{H}_{2} \mathrm{O}$; Lone Creek Fall Cave, South Africa

Löweite $-\mathrm{Na}_{12} \mathrm{Mg}_{7}\left(\mathrm{SO}_{4}\right)_{13} \cdot 15 \mathrm{H}_{2} \mathrm{O}$; Grillid volcanic cave, Surtsey Island, Iceland

Mascagnite - $\left(\mathrm{NH}_{4}\right)_{2} \mathrm{SO}_{4}$; Ruatapu Cave, New Zealand (Rodgers et al., 2000)

Melanterite $-\mathrm{FeSO}_{4} \cdot 7 \mathrm{H}_{2} \mathrm{O}$; Wilson Cave (?), Nevada, USA

Mendozite $-\mathrm{NaAl}\left(\mathrm{SO}_{4}\right)_{2} \cdot 11 \mathrm{H}_{2} \mathrm{O}$; Kitum Cave, Kenya (Forti et al., 2003)

Meta-aluminite $-\mathrm{Al}_{2} \mathrm{SO}_{4}(\mathrm{OH})_{4} \cdot 5 \mathrm{H}_{2} \mathrm{O}$; Valea Rea Cave, Romania (Feier, 2003)

Metavoltine $-\mathrm{K}_{2} \mathrm{Na}_{6} \mathrm{Fe}^{2+}\left(\mathrm{Fe}^{3+}\right)_{6} \mathrm{O}_{2}\left(\mathrm{SO}_{4}\right)_{12} \cdot 18 \mathrm{H}_{2} \mathrm{O}$; Alum Cave, Italy

Millosevichite $-\mathrm{Al}_{2}\left(\mathrm{SO}_{4}\right)_{3}$; Alum Cave, Italy

Mirabilite $-\mathrm{Na}_{2} \mathrm{SO}_{4} \cdot 10 \mathrm{H}_{2} \mathrm{O}$; cave type locality unknown

Misenite $-\mathrm{K}_{8}\left(\mathrm{SO}_{4}\right)\left(\mathrm{SO}_{3} \mathrm{OH}\right)_{6}$; Grotta dello Zolfo, Italy

Natroalunite - $\mathrm{NaAl}_{3}\left(\mathrm{SO}_{4}\right)_{2}(\mathrm{OH})_{6}$; Lechuguilla Cave, USA

Natrojarosite $-\mathrm{Na}\left(\mathrm{Fe}^{3+}\right)_{3}\left(\mathrm{SO}_{4}\right)_{2}(\mathrm{OH})_{6}$; Jungle Pot Cave, South Africa

Pickeringite $-\mathrm{MgAl}_{2}\left(\mathrm{SO}_{4}\right)_{4} \cdot 22 \mathrm{H}_{2} \mathrm{O}$; Grotta dello Zolfo, Italy

Picromerite $-\mathrm{K}_{2} \mathrm{Mg}\left(\mathrm{SO}_{4}\right)_{2} \cdot 6 \mathrm{H}_{2} \mathrm{O}$; Cutrona lava tube, Mt. Etna, Italy

Plumbojarosite $-\mathrm{Pb}_{0.5}\left(\mathrm{Fe}^{3+}\right)_{3}\left(\mathrm{SO}_{4}\right)_{2}(\mathrm{OH})_{6} ;+50$ Cave Naica, Mexico (Forti, 2010)

Polyhalite $-\mathrm{K}_{2} \mathrm{Ca}_{2} \mathrm{Mg}\left(\mathrm{SO}_{4}\right)_{4} \cdot 2 \mathrm{H}_{2} \mathrm{O}$; Cutrona lava tube, Mt. Etna, Italy 
Rapidcreekite $-\mathrm{Ca}_{2}\left(\mathrm{SO}_{4}\right)\left(\mathrm{CO}_{3}\right) \cdot 4 \mathrm{H}_{2} \mathrm{O}$; Diana Cave, Romania (Onac et al., 2009b)

Römerite $-\mathrm{Fe}^{2+}\left(\mathrm{Fe}^{3+}\right)_{2}\left(\mathrm{SO}_{4}\right)_{4} \cdot 14 \mathrm{H}_{2} \mathrm{O}$; Carlsbad Caverns, USA

Rozenite $-\mathrm{Fe}^{2+} \mathrm{SO}_{4} \cdot 4 \mathrm{H}_{2} \mathrm{O}$; Faggeto Tondo, Italy

Sabieite $-\mathrm{NH}_{4} \mathrm{Fe}^{3+}\left(\mathrm{SO}_{4}\right)_{2}$; Lone Creek Fall Cave, South Africa

Serpierite $-\mathrm{Ca}(\mathrm{Cu}, \mathrm{Zn})_{4}\left(\mathrm{SO}_{4}\right)_{2}(\mathrm{OH})_{6} \cdot 3 \mathrm{H}_{2} \mathrm{O}$; Cave \#4, Runcului Hill, Romania (Zaharia et al., 2003)

Siderotil - $(\mathrm{Fe}, \mathrm{Cu})\left(\mathrm{SO}_{4}\right) \cdot 5 \mathrm{H}_{2} \mathrm{O}$; Ruatapu Cave, New Zealand (Rodgers et al., 2000)

Spangolite $-\mathrm{Cu}_{6} \mathrm{AlSO}_{4}(\mathrm{OH})_{12} \mathrm{Cl} \cdot 3 \mathrm{H}_{2} \mathrm{O}$; Bingham mine caves, USA

Starkeyite $-\mathrm{MgSO}_{4} \cdot 4 \mathrm{H}_{2} \mathrm{O}$; Cueva de las Velas, Naica, Mexico (Forti et al., 2007)

Syngenite $-\mathrm{K}_{2} \mathrm{Ca}\left(\mathrm{SO}_{4}\right)_{2} \cdot \mathrm{H}_{2} \mathrm{O}$; Murra-el-elevyn Cave, Australia

Szmikite - $\mathrm{MnSO}_{4} \cdot \mathrm{H}_{2} \mathrm{O}$; Cueva de las Velas, Naica, Mexico (Forti et al., 2007)

Szmolnokite $-\mathrm{FeSO}_{4} \cdot \mathrm{H}_{2} \mathrm{O}$; Cueva de las Velas, Naica, Mexico (Forti et al., 2007)

Tamarugite $-\mathrm{NaAl}\left(\mathrm{SO}_{4}\right)_{2} \cdot 6 \mathrm{H}_{2} \mathrm{O}$; Grotta dello Zolfo, Italy

Thenardite $-\mathrm{Na}_{2} \mathrm{SO}_{4}$; Grotta delle Argille, Modena, Italy

Tschermigite $-\mathrm{NH}_{4} \mathrm{Al}\left(\mathrm{SO}_{4}\right)_{2} \cdot 12 \mathrm{H}_{2} \mathrm{O}$; Ruatapu Cave, New Zealand

Voltaite $-\mathrm{K}_{2}\left(\mathrm{Fe}^{2+}\right)_{5}\left(\mathrm{Fe}^{3+}\right)_{3} \mathrm{Al}\left(\mathrm{SO}_{4}\right)_{12} \cdot 18 \mathrm{H}_{2} \mathrm{O}$; Grotta dello Zolfo, Italy

Zaherite $-\mathrm{Al}_{12}\left(\mathrm{SO}_{4}\right)_{5}(\mathrm{OH})_{26} \cdot 20 \mathrm{H}_{2} \mathrm{O}$; Alum Cave, Italy (Forti et al., 1996)

\section{Cromates}

Crocoite - $\mathrm{PbCrO}_{4}$; Scărişoara Ice Cave, Romania (?) (Onac, 2001)

\section{Phosphates, Arsenates, Vanadates}

Phosphates

Archerite $-\mathrm{H}_{2} \mathrm{KPO}_{4}$; Petrogale Cave, Australia

Ardealite $-\mathrm{Ca}_{2}\left(\mathrm{PO}_{3} \mathrm{OH}\right)\left(\mathrm{SO}_{4}\right) \cdot 4 \mathrm{H}_{2} \mathrm{O}$; Cioclovina Cave, Romania

"Arnhemite" $-\mathrm{K}_{4} \mathrm{Mg}_{2}\left(\mathrm{P}_{2} \mathrm{O}_{7}\right) \cdot 5 \mathrm{H}_{2} \mathrm{O}$; Arnhem Cave, Namibia; Not approved by IMA-CNMNC

Berlinite - $\mathrm{AlPO}_{4}$; Cioclovina Cave; Romania (Onac et al., 2002)

Biphosphammite $-\mathrm{H}_{2}\left(\mathrm{NH}_{4}\right) \mathrm{PO}_{4}$; Murra-el-elevyn Cave, Australia

Bobierrite $-\mathrm{Mg}_{3}\left(\mathrm{PO}_{4}\right)_{3} \cdot 8 \mathrm{H}_{2} \mathrm{O}$; Imperial Canyon lava tubes, Kenya

Brushite $-\mathrm{Ca}\left(\mathrm{PO}_{3} \mathrm{OH}\right) \cdot 2 \mathrm{H}_{2} \mathrm{O}$; Skipton lava tubes, Australia

Carbonate-fluorapatite - Discredited by IMA-CNMNC

Carbonate-hydroxylapatite - Discredited by IMA-CNMNC

Chlorapatite $-\mathrm{Ca}_{5}\left(\mathrm{PO}_{4}\right)_{3} \mathrm{Cl}$; Lyon Cave, Philippines

Churchite-(Y) - $\mathrm{YPO}_{4} \cdot 2 \mathrm{H}_{2} \mathrm{O}$; Cioclovina Cave, Romania (Onac et al., 2005)

Collinsite $-\mathrm{Ca}_{2} \mathrm{Mg}\left(\mathrm{PO}_{4}\right)_{2} \cdot 2 \mathrm{H}_{2} \mathrm{O}$; Blue Lagoon Cave, South Africa

Crandallite $-\mathrm{CaAl}_{3}\left(\mathrm{PO}_{4}\right)_{2}\left(\mathrm{PO}_{3} \mathrm{OH}\right)(\mathrm{OH})_{6}$; caves on Isla Mona, Puerto Rico

Diadochite $-\left(\mathrm{Fe}^{3+}\right)_{2}\left(\mathrm{PO}_{4}\right)\left(\mathrm{SO}_{4}\right)(\mathrm{OH}) \cdot 6 \mathrm{H}_{2} \mathrm{O}$; Feengrotten, Germany

Dittmarite $-\left(\mathrm{NH}_{4}\right) \mathrm{MgPO}_{4} \cdot \mathrm{H}_{2} \mathrm{O}$; Skipton lava tubes, Australia

Evansite $-\mathrm{Al}_{3} \mathrm{PO}_{4}(\mathrm{OH})_{6} \cdot 6 \mathrm{H}_{2} \mathrm{O}$; sandstone cave in Columbia, SouthAmerica

Fluorapatite - $\mathrm{Ca}_{5}\left(\mathrm{PO}_{4}\right)_{3} \mathrm{~F}$; Slaughter Canyon Cave, New Mexico, SUA

Foggite $-\mathrm{CaAlPO}_{4}(\mathrm{OH})_{2} \cdot \mathrm{H}_{2} \mathrm{O}$; Cioclovina Cave, Romania (Onac et al., 2005)

Francoanellite $-\mathrm{K}_{3} \mathrm{Al}_{5}\left(\mathrm{PO}_{3} \mathrm{OH}\right)\left(\mathrm{PO}_{4}\right)_{2} \cdot 12 \mathrm{H}_{2} \mathrm{O}$; Castellana caves, Italy

Gordonite $-\mathrm{MgAl}_{2}\left(\mathrm{PO}_{4}\right)_{2}(\mathrm{OH})_{2} \cdot 8 \mathrm{H}_{2} \mathrm{O}$; Parwan Cave, Victoria, Asutralia

Hannayite - $\left(\mathrm{NH}_{4}\right)_{2} \mathrm{Mg}_{3}\left(\mathrm{PO}_{3} \mathrm{OH}\right)_{4} \cdot 8 \mathrm{H}_{2} \mathrm{O}$; Skipton lava tubes, Australia

Hopeite $-\mathrm{Zn}_{3}\left(\mathrm{PO}_{4}\right)_{2} \cdot 4 \mathrm{H}_{2} \mathrm{O}$ (orth.); Broken Hills mine caves, Zambia

Hydroxylapatite $-\mathrm{Ca}_{5}\left(\mathrm{PO}_{4}\right)_{3}(\mathrm{OH})$; caves on Isla Mona, Puerto Rico

Kingsmountite $-\mathrm{Ca}_{4} \mathrm{Fe}^{2+} \mathrm{Al}_{4}\left(\mathrm{PO}_{4}\right)_{6}(\mathrm{OH})_{4} \cdot 12 \mathrm{H}_{2} \mathrm{O}$; Rossillo Cave, Quatro Cienegas Desert, Mexico (Forti et al., 2006)

Koninckite $-\mathrm{Fe}^{3+} \mathrm{PO}_{4} \cdot 3 \mathrm{H}_{2} \mathrm{O}$; Oni-Ana Cave, Japan

Leucophosphite $-\mathrm{K}\left(\mathrm{Fe}^{3+}\right)_{2}\left(\mathrm{PO}_{4}\right)_{2}(\mathrm{OH}) \cdot 2 \mathrm{H}_{2} \mathrm{O}$; Bomi Hill caves, Liberia

Lipscombite $-\mathrm{Fe}^{2+}\left(\mathrm{Fe}^{3+}\right)_{2}\left(\mathrm{PO}_{4}\right)_{2}(\mathrm{OH})_{2}$; Perak Tong Cave, Malaysia

Minyulite - $\mathrm{KAl}_{2}\left(\mathrm{PO}_{4}\right)_{2} \mathrm{~F} \cdot 4 \mathrm{H}_{2} \mathrm{O}$; Boon Cave, Transvaal, South Africa

Mitridatite $-\mathrm{Ca}_{2}\left(\mathrm{Fe}^{3+}\right)_{3} \mathrm{O}_{2}\left(\mathrm{PO}_{4}\right)_{3} \cdot 3 \mathrm{H}_{2} \mathrm{O}$; Boon Cave, Transvaal, South Africa

Monetite $-\mathrm{Ca}\left(\mathrm{PO}_{3} \mathrm{OH}\right)$; caves on Isla Mona, Puerto Rico

Montgomeryite $-\mathrm{Ca}_{4} \mathrm{MgAl}_{4}\left(\mathrm{PO}_{4}\right)_{6}(\mathrm{OH})_{4} \cdot 12 \mathrm{H}_{2} \mathrm{O}$; et-Tabun Cave, Israel

Mundrabillaite $-\left(\mathrm{NH}_{4}\right)_{2} \mathrm{Ca}\left(\mathrm{PO}_{3} \mathrm{OH}\right)_{2} \cdot \mathrm{H}_{2} \mathrm{O}$; Petrogale Cave, W. Australia

Newberyite $-\mathrm{Mg}\left(\mathrm{PO}_{3} \mathrm{OH}\right) \cdot 3 \mathrm{H}_{2} \mathrm{O}$; Skipton lava tubes, Australia

Niahite $-\left(\mathrm{NH}_{4}\right) \mathrm{Mn}^{2+} \mathrm{PO}_{4} \cdot \mathrm{H}_{2} \mathrm{O}$; Niah Great Cave, Sarawak, Malaysia

Parahopeite $-\mathrm{Zn}_{3}\left(\mathrm{PO}_{4}\right)_{2} \cdot 4 \mathrm{H}_{2} \mathrm{O}$ (tric.); Hudson Bay mine caves, British Columbia, Canada

Phosphammite - $\left(\mathrm{NH}_{4}\right)_{2}\left(\mathrm{PO}_{3} \mathrm{OH}\right)$; Toppin Hill caves, Australia

Phosphosiderite $-\mathrm{Fe}^{3+}\left(\mathrm{PO}_{4}\right) \cdot 2 \mathrm{H}_{2} \mathrm{O}$; Bomi Hill caves, Liberia

Purpurite - $\left(\mathrm{Mn}^{3+}, \mathrm{Fe}^{3+}\right) \mathrm{PO}_{4}$; Gunong Keriang, Malaysia

"Pyrocoproite" - $(\mathrm{K}, \mathrm{Na})_{2} \mathrm{Mg}\left(\mathrm{P}_{2} \mathrm{O}_{7}\right)$; Arnhem Cave, Namibia; Not approved by IMA-CNMNC (Martini, 1997)

Pyromorphite - $\mathrm{Pb}_{5}\left(\mathrm{PO}_{4}\right)_{3} \mathrm{Cl}$; cave in Friedricksgessen mine, Germany

"Pyrophosphite" $-\mathrm{K}_{2} \mathrm{CaP}_{2} \mathrm{O}_{7}$; Arnhem Cave, Namibia; Not approved by IMA-CNMNC

Studia UBB Geologia, 2011, 56 (1), 33 - 42 
Sampleite - $\mathrm{NaCaCu}_{5}\left(\mathrm{PO}_{4}\right)_{4} \mathrm{Cl} \cdot 5 \mathrm{H}_{2} \mathrm{O}$; Mbobo Mkulu Cave, Transvaal, South Africa

Sasaite $-\mathrm{Al}_{6}\left(\mathrm{PO}_{4}\right)_{5}(\mathrm{OH})_{3} \cdot 36 \mathrm{H}_{2} \mathrm{O}$; West Driefontein Cave, South Africa

Schertelite $-\left(\mathrm{NH}_{4}\right)_{2} \mathrm{Mg}\left(\mathrm{PO}_{3} \mathrm{OH}\right)_{2} \cdot 4 \mathrm{H}_{2} \mathrm{O}$; Skipton lava tubes, Australia

Scholzite $-\mathrm{CaZn}_{2}\left(\mathrm{PO}_{4}\right)_{2} \cdot 2 \mathrm{H}_{2} \mathrm{O}$; cave in Virginia, USA

Spencerite $-\mathrm{Zn}_{4}\left(\mathrm{PO}_{4}\right)_{2}(\mathrm{OH})_{2} \cdot 3 \mathrm{H}_{2} \mathrm{O}$; Hudson Bay mine caves, British Columbia, Canada

Stercorite $-\left(\mathrm{NH}_{4}\right) \mathrm{Na}\left(\mathrm{PO}_{3} \mathrm{OH}\right) \cdot 4 \mathrm{H}_{2} \mathrm{O}$; Petrogale Cave, W. Australia

Strengite $-\mathrm{Fe}^{3+} \mathrm{PO}_{4} \cdot 2 \mathrm{H}_{2} \mathrm{O}$; Bomi Hill caves, Liberia

Struvite - $\left(\mathrm{NH}_{4}\right) \mathrm{MgPO}_{4} \cdot 6 \mathrm{H}_{2} \mathrm{O}$; Skipton lava tubes, Australia

Swaknoite - $\left(\mathrm{NH}_{4}\right)_{2} \mathrm{Ca}\left(\mathrm{PO}_{3} \mathrm{OH}\right)_{2} \cdot \mathrm{H}_{2} \mathrm{O}$; Arnhem Cave, Namibia

Taranakite $-\mathrm{K}_{3} \mathrm{Al}_{5}\left(\mathrm{PO}_{3} \mathrm{OH}\right)_{6}\left(\mathrm{PO}_{4}\right)_{2} \cdot 18 \mathrm{H}_{2} \mathrm{O}$; Minerva Cave, France

Tarbuttite $-\mathrm{Zn}_{2} \mathrm{PO}_{4}(\mathrm{OH})$; Broken Hills mine caves, Zambia

Tinsleyite $-\mathrm{KAl}_{2}\left(\mathrm{PO}_{4}\right)_{2}(\mathrm{OH}) \cdot 2 \mathrm{H}_{2} \mathrm{O}$; Cioclovina Cave, Romania (Marincea et al., 2002)

Tinticite $-\left(\mathrm{Fe}^{3+}\right)_{5.3}\left(\mathrm{PO}_{4}\right)_{4}(\mathrm{OH})_{6} \cdot 6.7 \mathrm{H}_{2} \mathrm{O}$; cavity in the Tintic district, UT, USA

Variscite $-\mathrm{AlPO}_{4} \cdot 2 \mathrm{H}_{2} \mathrm{O}$; Drachenhöhle Cave, Austria

Vashegyite - $\mathrm{Al}_{11}\left(\mathrm{PO}_{4}\right)_{9}(\mathrm{OH})_{6} \cdot 38 \mathrm{H}_{2} \mathrm{O}$; Oni-Ana; Tateishi-Shônyû-dô caves, Japan

Vivianite $-\left(\mathrm{Fe}^{2+}\right)_{3}\left(\mathrm{PO}_{4}\right)_{2} \cdot 8 \mathrm{H}_{2} \mathrm{O}$; Niah Great Cave, Sarawak, Malaysia

Wavellite $-\mathrm{Al}_{3}\left(\mathrm{PO}_{4}\right)_{2}(\mathrm{OH})_{3} \cdot 5 \mathrm{H}_{2} \mathrm{O}$; Valea Rea Cave, Romania

Whitlockite $-\mathrm{Ca}_{9} \mathrm{Mg}\left(\mathrm{PO}_{3} \mathrm{OH}\right)\left(\mathrm{PO}_{4}\right)_{6}$; El Chapote Cave, Mexico

Woodhouseite $-\mathrm{CaAl}_{3}\left(\mathrm{SO}_{4}\right)\left(\mathrm{PO}_{4}\right)(\mathrm{OH})_{6}$; Jade Lotus Cave, Yangshuo, China

\section{Arsenates}

Arseniosiderite $-\mathrm{Ca}_{2}\left(\mathrm{Fe}^{3+}\right)_{3} \mathrm{O}_{2}\left(\mathrm{AsO}_{4}\right)_{3} \cdot 3 \mathrm{H}_{2} \mathrm{O}$; Tyuya-Muyun Cave, Kyrgyzstan

Beudantite $-\mathrm{Pb}\left(\mathrm{Fe}^{3+}\right)_{3}\left(\mathrm{AsO}_{4}\right)\left(\mathrm{SO}_{4}\right)(\mathrm{OH})_{6}$; Island Ford Cave, USA

Conichalcite - $\mathrm{CaCuAsO}_{4}(\mathrm{OH})$; Corkscrew Cave, Grand Canyon, USA

Hedyphane $-\mathrm{Ca}_{2} \mathrm{~Pb}_{3}\left(\mathrm{AsO}_{4}\right)_{3} \mathrm{Cl}$; cave in Santa Barbara mine, Sardinia, Italy (De Waele and Forti, 2005)

Hörnesite - $\mathrm{Mg}_{3}\left(\mathrm{AsO}_{4}\right)_{2} \cdot 8 \mathrm{H}_{2} \mathrm{O}$; Corkscrew Cave, Grand Canyon, USA (not cited in CMW2) (Wenrich and Sutphin, 1994)

Manganberzeliite - $\mathrm{NaCa}_{2}\left(\mathrm{Mn}^{2+}\right)_{2}\left(\mathrm{AsO}_{4}\right)_{3}$; Cueva Alfredo Jahn, Venezuela

Mimetite $-\mathrm{Pb}_{5}\left(\mathrm{AsO}_{4}\right)_{3} \mathrm{Cl}$; Bisbee mine cave, $\mathrm{AZ}$, USA

Olivenite $-\mathrm{Cu}_{2} \mathrm{AsO}_{4}(\mathrm{OH})$; cavities in the Tintic district, UT, USA

Pharmacolite $-\mathrm{Ca}\left(\mathrm{AsO}_{3} \mathrm{OH}\right) \cdot 2 \mathrm{H}_{2} \mathrm{O}$; Corkscrew Cave, Grand Canyon, USA (Onac et al., 2007b)

Strashimirite $-\mathrm{Cu}_{4}\left(\mathrm{AsO}_{4}\right)_{2}(\mathrm{OH})_{2} \cdot 2.5 \mathrm{H}_{2} \mathrm{O}$; Dupkata na Mara Cave, Bulgaria

Talmessite $-\mathrm{Ca}_{2} \mathrm{Mg}\left(\mathrm{AsO}_{4}\right)_{2} \cdot 2 \mathrm{H}_{2} \mathrm{O}$; Corkscrew Cave, Grand Canyon, USA

Yukonite $-\mathrm{Ca}_{7}\left(\mathrm{Fe}^{3+}\right)_{15}\left(\mathrm{AsO}_{4}\right)_{9} \mathrm{O}_{16} \cdot 25 \mathrm{H}_{2} \mathrm{O}$ (?); Grotta della Monaca, Italy (Garavelli et al., 2009)

\section{Vanadates}

Calciovolborthite - Discredited by IMA-CNMNC (use tangeite)

Carnotite $-\mathrm{K}_{2}\left(\mathrm{UO}_{2}\right)_{2}\left(\mathrm{VO}_{4}\right)_{2} \cdot 3 \mathrm{H}_{2} \mathrm{O}$; Tyuya-Muyun Cave, Kyrgyzstan

Descloizite $-\mathrm{PbZnVO}_{4}(\mathrm{OH})$; Tyuya-Muyun Cave, Kyrgyzstan

Metatyuyamunite $-\mathrm{Ca}\left(\mathrm{UO}_{2}\right)_{2}\left(\mathrm{VO}_{4}\right)_{2} \cdot 3 \mathrm{H}_{2} \mathrm{O}$; Spider Cave, New Mexico, USA

Tangeite $-\mathrm{CaCuVO}_{4}(\mathrm{OH})$; Zelania Cave, Tyuya-Muyun, Kyrgyzstan

Tyuyamunite $-\mathrm{Ca}\left(\mathrm{UO}_{2}\right)_{2}\left(\mathrm{VO}_{4}\right)_{2} \cdot 3 \mathrm{H}_{2} \mathrm{O}$; Tyuya-Muyun Cave, Kyrgyzstan

Vanadinite $-\mathrm{Pb}_{5}\left(\mathrm{VO}_{4}\right)_{3} \mathrm{Cl}$; Broken Hills mine caves, Zambia

\section{Molybdates}

Powellite - $\mathrm{CaMoO}_{4}$; Corkscrew Cave, Grand Canyon, USA (not cited in CMW2) (Wenrich and Sutphin, 1994)

\section{Organic compounds}

Acetamide $-\mathrm{CH}_{3} \mathrm{CONH}_{2}$; Prilepnata Cave, Bulgaria

Glushinskite $-\mathrm{MgC}_{2} \mathrm{O}_{4} \cdot 2 \mathrm{H}_{2} \mathrm{O}$; Temple of Dome Cave, Namibia

Guanine $-\mathrm{C}_{5} \mathrm{H}_{3}\left(\mathrm{NH}_{2}\right) \mathrm{N}_{4} \mathrm{O}$; Murra-el-elevyn Cave, Australia

Mellite $-\mathrm{Al}_{2} \mathrm{C}_{6}(\mathrm{COO})_{6} \cdot 16 \mathrm{H}_{2} \mathrm{O}$; Romanelli Cave, Apulia, Italy

Oxammite $-\left(\mathrm{NH}_{4}\right)_{2} \mathrm{C}_{2} \mathrm{O}_{4} \cdot \mathrm{H}_{2} \mathrm{O}$; Petrogale Cave, W. Australia

Urea - $\mathrm{CO}\left(\mathrm{NH}_{2}\right)_{2}$; Wilgie Mia Cave, W. Australia

Uricite $-\mathrm{C}_{5} \mathrm{H}_{4} \mathrm{~N}_{4} \mathrm{O}_{3}$; Dingo Donga Cave, W. Australia

Weddellite $-\mathrm{CaC}_{2} \mathrm{O}_{4} \cdot 2 \mathrm{H}_{2} \mathrm{O}$; Toppin Hill Cave, W. Australia

Whewellite $-\mathrm{CaC}_{2} \mathrm{O}_{4} \cdot \mathrm{H}_{2} \mathrm{O}$; Parakietgat Cave, Namibia

\section{Silicates}

Allophane - $\mathrm{Al}_{2} \mathrm{O}_{3}\left(\mathrm{SiO}_{2}\right)_{1.3-2.0} \cdot 2.5-3 \mathrm{H}_{2} \mathrm{O}$; Tyuya-Muyun Cave, Kyrgyzstan

Apophyllite-(KOH) - $\mathrm{KCa}_{4} \mathrm{Si}_{8} \mathrm{O}_{20}(\mathrm{OH}, \mathrm{F}) \cdot 8 \mathrm{H}_{2} \mathrm{O}$; Kitum Cave, Kenya (Forti et al., 2003)

Benitoite - $\mathrm{BaTiSi}_{3} \mathrm{O}_{9}$; Iza Cave, Romania

Boltwoodite $-\mathrm{KUO}_{2}\left(\mathrm{SiO}_{3} \mathrm{OH}\right) \cdot \mathrm{H}_{2} \mathrm{O}$; cave in northern Chihuahua, Mexico

Chrysocolla - $(\mathrm{Cu}, \mathrm{Al})_{2} \mathrm{H}_{2} \mathrm{Si}_{2} \mathrm{O}_{5}(\mathrm{OH})_{4} \cdot \mathrm{nH}_{2} \mathrm{O}$; Tyuya-Muyun Cave, Kyrgyzstan 
Clinochlore $-\mathrm{Mg}_{6} \mathrm{Si}_{4} \mathrm{O}_{10}(\mathrm{OH})_{8}$; Monte Rosso Cave, Reggio Emilia, Italy

Clinoptilolite- $\mathrm{Na}-\mathrm{Na}_{6}\left(\mathrm{Si}_{30} \mathrm{Al}_{6}\right) \mathrm{O}_{72} \cdot 20 \mathrm{H}_{2} \mathrm{O}$; Cave in the Culachao mine, Chile (De Waele et al., 2009)

Dickite $-\mathrm{Al}_{2} \mathrm{Si}_{2} \mathrm{O}_{5}(\mathrm{OH})_{4}$; Iza Cave, Romania

Endellite - Discredited by IMA-CNMNC (use Halloysite-10A)

Epidote $-\mathrm{Ca}_{2} \mathrm{Fe}^{3+} \mathrm{Al}_{2}\left(\mathrm{Si}_{2} \mathrm{O}_{7}\right)\left(\mathrm{SiO}_{4}\right) \mathrm{O}(\mathrm{OH})$; Santo Cave, Mt. Etna, Italy

Fraipontite - $(\mathrm{Zn}, \mathrm{Al})_{3}(\mathrm{Si}, \mathrm{Al})_{2} \mathrm{O}_{5}(\mathrm{OH})_{4}$; Cupp-Coutunn Cave, Turkmenistan

Halloysite- $7 \AA-\mathrm{Al}_{2} \mathrm{Si}_{2} \mathrm{O}_{5}(\mathrm{OH})_{4}$ (mon.); Faggeto Tondo Cave, Italy

Halloysite- $10 \AA-\mathrm{Al}_{2} \mathrm{Si}_{2} \mathrm{O}_{5}(\mathrm{OH})_{4} \cdot 2 \mathrm{H}_{2} \mathrm{O}$; caves in the Guadalupe Mountains, USA

Hectorite $-\mathrm{Na}_{0.3}(\mathrm{Mg}, \mathrm{Li})_{3} \mathrm{Si}_{4} \mathrm{O}_{10}(\mathrm{~F}, \mathrm{OH})_{2} \cdot n \mathrm{H}_{2} \mathrm{O}$; Cueva de las Espadas, Naica, Mexico (Forti et al., 2009)

Hemimorphite $-\mathrm{Zn}_{4} \mathrm{Si}_{2} \mathrm{O}_{7}(\mathrm{OH})_{2} \cdot \mathrm{H}_{2} \mathrm{O}$; Hudson Bay mine caves, Canada

Howlite $-\mathrm{Ca}_{2} \mathrm{SiB}_{5} \mathrm{O}_{9}(\mathrm{OH})_{5}$; Ordinskaya Cave, Kungur, Russia (Potapov and Parshima, 2010)

Hydroxyapophyllite - Discredited by IMA-CNMNC [use Apophyllite-(KOH)]

Hydroxylellestadite $-\mathrm{Ca}_{10}\left(\mathrm{SiO}_{4}\right)_{3}\left(\mathrm{SO}_{4}\right)_{3}(\mathrm{OH})_{2}$; Cioclovina Cave, Romania (Onac et al., 2006)

Illite - NOT a mineral; Name used to designate a group of species

Ilvaite $-\mathrm{CaFe}^{3+}\left(\mathrm{Fe}^{2+}\right)_{2} \mathrm{O}\left(\mathrm{Si}_{2} \mathrm{O}_{7}\right)(\mathrm{OH})$; hydrothermal skarn caves at Primarsky Kray, Russia

Kaolinite $-\mathrm{Al}_{2} \mathrm{Si}_{2} \mathrm{O}_{5}(\mathrm{OH})_{4}$ (tric.); Cupp-Coutunn Cave, Turkmenistan

Montmorillonite - $\left.\left.(\mathrm{Na}, \mathrm{Ca})_{0.3}(\mathrm{Al}, \mathrm{Mg})_{2}\right) \mathrm{Si}_{4} \mathrm{O}_{10}\right)(\mathrm{OH})_{2} \cdot n \mathrm{H}_{2} \mathrm{O}$; Carlsbad Caverns, USA

Nacrite $-\mathrm{Al}_{2} \mathrm{Si}_{2} \mathrm{O}_{5}(\mathrm{OH})_{4}$ (mon.); Valea Rea Cave, Romania (Ghergari and Tămaş, 1996)

Natrolite $-\mathrm{Na}_{2}\left(\mathrm{Si}_{3} \mathrm{Al}_{2}\right) \mathrm{O}_{10} \cdot 2 \mathrm{H}_{2} \mathrm{O}$; Big Cave from Bolfu mine III, Romania

Nontronite $-\mathrm{Na}_{0.3}\left(\mathrm{Fe}^{3+}\right)_{2}(\mathrm{Si}, \mathrm{Al})_{4} \mathrm{O}_{10}(\mathrm{OH})_{2} \cdot n \mathrm{H}_{2} \mathrm{O}$; Kartchner Caverns, USA

Orientite $-\mathrm{Ca}_{8}\left(\mathrm{Mn}^{3+}\right)_{10}\left(\mathrm{SiO}_{4}\right)_{3}\left(\mathrm{Si}_{3} \mathrm{O}_{10}\right)_{3}(\mathrm{OH})_{10} \cdot 4 \mathrm{H}_{2} \mathrm{O}$; Cueva de las Velas, Naica, Mexico (Forti et al., 2007)

Palygorskite - $(\mathrm{Mg}, \mathrm{Al})_{2} \mathrm{Si}_{4} \mathrm{O}_{10}(\mathrm{OH}) \cdot 4 \mathrm{H}_{2} \mathrm{O}$; Broken Hill caves, New Zealand

Phillipsite-K $-\mathrm{K}_{6}\left(\mathrm{Si}_{10} \mathrm{Al}_{6}\right) \mathrm{O}_{32} \cdot 12 \mathrm{H}_{2} \mathrm{O}$; Kitum Cave, Kenya (Forti et al., 2003)

Rectorite $-(\mathrm{Na}, \mathrm{Ca}) \mathrm{Al}_{4}(\mathrm{Si}, \mathrm{Al})_{8} \mathrm{O}_{20}(\mathrm{OH})_{4} \cdot 2 \mathrm{H}_{2} \mathrm{O}$; Kartchner Caverns, USA

Saponite - $(\mathrm{Ca}, \mathrm{Na})_{0.3}(\mathrm{Mg}, \mathrm{Fe})_{3}(\mathrm{Si}, \mathrm{Al})_{4} \mathrm{O}_{10}(\mathrm{OH})_{2} \cdot 4 \mathrm{H}_{2} \mathrm{O}$; Vântului Cave, Romania

Sauconite $-\mathrm{Na}_{0.3} \mathrm{Zn}_{3}(\mathrm{Si}, \mathrm{Al})_{4} \mathrm{O}_{10}(\mathrm{OH})_{2} \cdot 4 \mathrm{H}_{2} \mathrm{O}$; Cupp-Coutunn Cave, Turkmenistan

Sepiolite $-\mathrm{Mg}_{4} \mathrm{Si}_{6} \mathrm{O}_{15}(\mathrm{OH})_{2} \cdot 6 \mathrm{H}_{2} \mathrm{O}$; Zbrasov Cave, Czech Republic

Shattuckite $-\mathrm{Cu}_{5}\left(\mathrm{SiO}_{3}\right)_{4}(\mathrm{OH})_{2}$; Bisbee mine caves, USA

Acknowledgements. We are grateful to all members of the Cave Minerals Commission of the International Union of Speleology who provided us with news and suggestions. This note is part of the Cave Mineral Database (CAMIDA) project, a collaborative effort of the University of South Florida Libraries Karst Information Portal, UIS's Cave Minerals Commission, the "Emil Racoviță" Institute of Speleology (Romania), and the Karst Research Group at the University of South Florida (USA).

\section{R E F E R E N C E S}

Audra, P. 2007, A mineralized hypogenic cave in Pierre Saint-Martin massif: the Oilloki Cave (Sainte-Engrâce, Pyrénées-Atlantiques). Preliminary investigations. Karstologia Memoire, 17: 176-182.

Audra, P., Hobléa, F. 2007, The first cave occurrence of jurbanite $\left[\mathrm{Al}\left(\mathrm{OH} \quad \mathrm{SO}_{4}\right) \cdot 5 \mathrm{H}_{2} \mathrm{O}\right]$, associated with alunogen $\left[\mathrm{Al}\left(\mathrm{SO}_{4}\right)_{3} \cdot 17 \mathrm{H}_{2} \mathrm{O}\right]$ and tschermigite $\left[\mathrm{NH}_{4} \mathrm{Al}\left(\mathrm{SO}_{4}\right)_{2} \cdot 12 \mathrm{H}_{2} \mathrm{O}\right]$ : thermal-sulfidic Serpents Cave, France. Journal of Cave and Karst Studies, 69 (2): 243- 249.

Burke, E.A.J. 2006, A mass discreditation of GQN minerals. Canadian Minerologist, 44: 1557-1560; DOI: http://dx.doi.org/10.2113/gscanmin.44.6.1557

Burke, E.A.J. 2008, Tidying up mineral names: an IMACNMNC scheme for suffixies, hyphens and diacritical marks. The Mineralogical Record 39: 131-135.

De Waele, J., Forti, P. 2005, Mineralogy of mine caves in Sardinia. Proceedings of the $14^{\text {th }}$ International Congress of Speleology, Kalamos, Greece, 306-311.

De Waele, J., Forti, P., Picotti, V., Galli, E., Rossi, A., Brook, G., Zini, L. \& Cucchi, F. 2009, Cave deposits

Studia UBB Geologia, 2011, 56 (1), 33 - 42 in Cordillera de la Sal (Atacama, Chile). In: Rossi P.L. (Ed.), Geological Constrains on the Onset and Evolution of an Extreme Environment: the Atacama area. Geoacta, Special Publication 2: 113-117.

Feier, N. 2003, New data on the mineralogy of Valea Rea Cave, Bihor Mountains. Ecocarst, 4: 22-24 (in Romanian).

Forti, P. 2002, Speleology in the third millenium: achievments and challenges. Theoretical and Applied Karstology, 15: 7-26.

Forti, P. 2005, Genetic processes of cave minerals in volcanic environment: an overview. Journal of Cave and Karst Studies, 67 (1): 3-13.

Forti, P. 2010, Genesis and evolution of the caves in the Naica Mine (Chihuahua, Mexico). Zeitschrift für Geomorphologie, 54 (2): 115-135; DOI: http://dx.doi.org/ 10.1127/0372-8854/2010/0054S2-0007

Forti, P., Galli, E. \& Rossi, A. 2003, Minerogenesis in some volcanic caves of Kenya. International Journal of Speleology, 32 (1/4): 1-16.

Forti, P., Galli, E. \& Rossi, A. 2006, Peculiar minerogenetic cave environments of Mexico: the Cuatro Ciénegas area. Acta Carsologica, 35 (2): 79-98.

Forti, P., Galli, E. \& Rossi, A. 2007, The mineralogical study on the Cueva de las Velas (Naica, Mexico). Acta Carsologica, 36 (3): 379-388.

Forti, P., Galli, E. \& Rossi, A. 2009, Minerogenesis in the Naica caves (Chihuahua, México). Proceedings of the $15^{\text {th }}$ International Congress of Speleology, Kerrville, 1: 300-305.

Forti, P., Panzica La Manna, M. \& Rossi, A. 1996, The peculiar mineralogical site of the Alum cave (Vulcano, Sicily). Proceedings of the $7^{\text {th }}$ International Symposium on Vulcanospeleology, Canarie, 35-44. 
Fridvaldszky, J. 1767, Mineralogia Magni Principatus Transylvaniae. Claudiopoli, Typis Academicis Societatis Jesu, $206 \mathrm{p}$ (in Latin).

Gaines, R.V., Skinner, H.C.W., Foord, E.E., Mason, B. \& Rosenzweig, A. 1997, Dana's New Mineralogy. Wiley, New York, 1819 p.

Garavelli, A., Pinto, D., Vurro, F., Mellini, M., Viti, C., Balic-Zunic, T. \& Della Ventura, G. 2009, Yukonite from the Grotta della Monaca Cave, Sant'Agata di Esaro, Italy: characterization and comparison with cotype material from the Daulton Nine, Yukon, Canada. Canadian Mineralogist, 47: 39-51; DOI: http://dx.doi.org/10.3749/canmin.47.1.39

Ghergari, L., Tămaş, T. 1996, Mineralogy of cave deposits from Bihor Mountains (Romania). In: Contribucion del studio cientifica de las cavidades kársticas al conocimiento geológico (Andreo, B., Carrasco, F. \& Duran J.J., Eds.), Patronato de la Cueva de Nerja, Nerja, p. 243-255.

Ghergari, L., Tămaş, T., Damm, P. \& Forray, F. 1997, Hydrothermal paleokarst in Pestera din Valea Rea (Bihor Mountains, Romania). Theoretical and Applied Karstology, 10: 115-125.

Hill, C.A. 1976, Cave minerals. National Speleological Society, Huntsville, 167 p.

Hill, C.A., Forti, P. 1986, Cave minerals of the world $\left(1^{\text {st }}\right.$ ed.). National Speleological Society, Huntsville, $238 \mathrm{p}$.

Hill, C.A., Forti, P. 1997, Cave minerals of the world $\left(2^{\text {nd }}\right.$ ed.). National Speleological Society, Huntsville, 464 p.

Hill, C.A., Forti, P. 2007, Cave mineralogy and the NSS: Past, present, and future. Journal of Cave and Karst Studies, 69 (1): 35-45.

Lazarides, G., Melfos, V. \& Papadopoulou, L. 2011, The first cave occurrence of orpiment $\left(\mathrm{As}_{2} \mathrm{~S}_{3}\right)$ from the sulfuric acid caves of Aghia Paraskevi (Kassandra Peninsula, N. Greece). International Journal of Speleology, 40 (2): in press

Marincea, Ş., Dumitraş, D. \& Gibert, R. 2002, Tinsleyite in the "dry" Cioclovina Cave (Şureanu Mountains, Romania): the second occurrence. European Journal of Mineralogy 14: 157-164; DOI: http://dx.doi.org/10.1127/ 0935-1221/2002/0014-0157

Martini, J.E.J. 1997, Pyrocoproite $\left(\mathrm{Mg}(\mathrm{K}, \mathrm{Na})_{2}\left(\mathrm{P}_{2} \mathrm{O}_{7}\right)\right.$, monoclinic) a new mineral from Arnhem Cave (Namibia), derived from bat guano combustion. Proceedings of the $12^{\text {th }}$ International Congress of Speleology, Le Chaux-de-Fonds, 1: 223-225.

Merino, A., Fornós, J.J. \& Onac, B.P. 2009, Preliminary data on mineralogical aspects of cave rims and vents in Cova des Pas de Vallgornera, Mallorca. Proceedings of the $15^{\text {th }}$ International Congress of Speleology, Kerrville, 1: 307-311.

Mills, S.J., Hatert, F., Nickel, E.H. \& Ferraris, G. 2009, The standardisation of mineral group hierarchies: application to recent nomenclature proposals. European Journal of Mineralogy, 21: 1073-1080; DOI: http://dx.doi.org/10.1127/0935-1221/2009/0021-1994

Moore, G.W. 1970, Checklist of cave minerals. National Speleological Society News, 28: 9-10.

Nickel, E.H., Grice, J.D. 1998, The IMA Commission on New Minerals and Mineral Names: procedures and guidelines on mineral nomenclature, 1998. Mineralogy and Petrology, 64 (1-4): 237-263; DOI: http://dx.doi.org/10.1007/BF01226571

Nickel, E.H., Nichols, M.C. 2009, IMA/CNMNC list of mineral names (http://pubsites.uws.edu.au/ima-cnmnc/ imalist.htm)

Onac, B.P. 2001, Mineralogical studies and Uraniumseries dating of speleothems from Scărişoara Glacier Cave (Bihor Mountains, Romania). Theoretical and Applied Karstology, 13-14: 33-38.

Onac, B.P. 2002, Caves formed within Upper Cretaceous skarns at Băița, Bihor County, Romania: mineral deposition and speleogenesis. Canadian Mineralogist, 40 (6): 1693-1703.

Onac, B.P. 2003, Minerals of the Carpathians: first update. Acta Mineralogica-Petrographica, 44: 31-34.

Onac, B.P. 2005, Minerals. In: Encyclopedia of caves (Culver D.C., White W.B., Eds.), Academic Press, New York, 371-378.

Onac B.P. 2008, Ikaite in the Scărişoara ice deposit: precipitation and significance. In: Proceedings $3^{\text {rd }}$ International Workshop on Ice Caves (Turri, S., Ed.), Perm State University, Perm, p. 28.

Onac, B.P. 2011, Minerals. In: Encyclopedia of caves $\left(2^{\text {nd }}\right.$ ed.) (Culver D.C., White W.B., Eds.), accepted.

Onac, B.P., Effenberger, H.S. 2007, Re-examination of berlinite $\left(\mathrm{AlPO}_{4}\right)$ from the Cioclovina Cave, Romania. American Mineralogist, 92: 1998-2001; DOI: http://dx.doi.org/10.2138/am.2007.2581

Onac, B.P., Forti, P. 2011, Minerogenetic mechanisms occurring in the cave environment: an overview. International Journal of Speleology, 40 (2): 1-20.

Onac, B.P., White, W.B. 2003, First reported sedimentary occurrence of berlinite $\left(\mathrm{AlPO}_{4}\right)$ in the phosphatebearing sediments from Cioclovina Cave, Romania. American Mineralogist, 88: 1395-1397.

Onac, B.P., Bernhardt, H.-J. \& Effenberger, H.S. 2009a, Authigenic burbankite in the Cioclovina Cave sediments (Romania). European Journal of Mineralogy, 21: 507514; DOI: http://dx.doi.org/10.1127/0935-1221/2009/ 0021-1916

Onac, B.P., Effenberger, H.S. \& Breban, R.C. 2007a, High-temperature and "exotic" minerals from the Cioclovina Cave, Romania: a review. Studia $U B B$ Geologia, 52 (2): 3-10; DOI: http://dx.doi.org/10.5038/ 1937-8602.52.2.1

Onac, B.P., Hess, J.W. \& White, W.B. 2007b, The relationship between the mineral composition of speleothems and mineralization of breccia pipes: evidence from Corkscrew Cave, Arizona, USA. Canadian Mineralogist, 45: 1177-1188; DOI: http://dx.doi.org/10.2113/gscanmin.45.5.1177

Onac, B.P., Mylroie, J.E. \& White, W.B. 2001a, Mineralogy of cave deposits on San Salvador Island, Bahamas. Carbonates and Evaporites, 16 (1): 8-16.

Onac, B.P., Pedersen, R.B. \& Tysseland, M. 1997, Presence of rare-earth elements in black ferromanganese coatings from Vântului Cave (Romania). Journal of Caves and Karst Studies, 59 (3): 128-131.

Onac, B.P., White, W.B. \& Viehmann, I. 2001b, Leonite $\mathrm{K}_{2} \mathrm{Mg}\left[\mathrm{SO}_{4}\right]_{2} \quad 4 \mathrm{H}_{2} \mathrm{O}$, konyaite $\mathrm{Na}_{2} \mathrm{Mg}\left[\mathrm{SO}_{4}\right]_{2} \quad 5 \mathrm{H}_{2} \mathrm{O}$ and syngenite $\mathrm{K}_{2} \mathrm{Ca}\left[\mathrm{SO}_{4}\right]_{2} \quad \mathrm{H}_{2} \mathrm{O}$ from Tăuşoare Cave (Rodnei Mts., Romania). Mineralogical Magazine, 65 (1): 1-7. 
Onac, B.P., Breban, R., Kearns, J. \& Tămaş, T. 2002, Unusual minerals related to phosphate deposits in Cioclovina Cave, Sureanu Mts. (Romania). Theoretical and Applied Karstology, 15: 27-34.

Onac, B.P., Effenberger, H., Ettinger, K. \& Cînta-Pînzaru, S. 2006, Hydroxylellestadite from Cioclovina Cave (Romania): Microanalytical, structural, and vibrational spectroscopy data. American Mineralogist, 91: 19271931; DOI: http://dx.doi.org/10.2138/am.2006.2143

Onac, B.P., Ettinger, K., Kearns, J. \& Balasz, I.I. 2005, A modern, guano-related occurrence of foggite, $\mathrm{CaAl}\left(\mathrm{PO}_{4}\right)(\mathrm{OH})_{2} \cdot \mathrm{H}_{2} \mathrm{O}$ and churchite-(Y), $\mathrm{YPO}_{4} \cdot 2 \mathrm{H}_{2} \mathrm{O}$ in Cioclovina Cave, Romania. Mineralogy and Petrology, 85: 291-302; DOI: http://dx.doi.org/ 10.1007/s00710-005-0106-4

Onac, B.P., Effenberger, H.S., Collins, N.C., Kearns, J.B. \& Breban, R.C. 2011, Revisiting three minerals from Cioclovina Cave (Romania). International Journal of Speleology, 40 (2): 21-30.

Onac, B.P., Sumrall, J.B., Tămaş, T., Povară, I., Kearns, J., Dârmiceanu, V., Vereş, D. \& Lascu, C. 2009b, The relationship between cave minerals and $\mathrm{H}_{2} \mathrm{~S}$-rich thermal waters along the Cerna Valley (SW Romania). Acta Carsologica, 38: 67-79.

Pasero, M., Kampf, A.R., Ferraris, C., Pekov, I.V., Rakovan, J. \& White, T.J. 2010, Nomenclature of the apatite supergroup minerals. European Journal of Mineralogy, 22: 163-179; DOI: http://dx.doi.org/ 10.1127/0935-1221/2010/0022-2022

Polyak, V.J., Provencio, P. 2001, By-product materials related to $\mathrm{H}_{2} \mathrm{~S}-\mathrm{H}_{2} \mathrm{SO}_{4}$ influenced speleogenesis of Carlsbad, Lechuguilla, and other caves of the Guadalupe Mountains, New Mexico. Journal of Cave and Karst Studies, 63: 23-32.
Potapov, S.S., Parshina, N.V. 2010, Howlite $\mathrm{Ca}_{2} \mathrm{~B}_{5} \mathrm{SiO}_{9}(\mathrm{OH})_{5}$ from Ordinskaya cave in Perm region - the first find on the Ural. In: Proceedings of Scientific Readings in Memory of P.N. Chirvinsky on Problems of Mineralogy, Petrography and Metallogeny. Perm State University, 13: 83-91 (in Russian).

Rodgers, K.A., Hamlin, K.A., Browne, P.R.L., Campbell, K.A. \& Martin, R. 2000, The steam condensate alteration mineralogy of Ruatapu cave, Orakei Korako geothermal field, Taupo Volcanic Zone, New Zealand. Mineralogical Magazine, 64 (1): 125-142.

Tămaş, T., Ghergari, L. 2003, Hydronium jarosite from Iza Cave (Rodnei Mts., Romania). Acta MineralogicaPetrographica Abstract Series, 1: 102.

Zaharia, L., Tămaş, T. \& Suciu-Krausz, E. 2003, Mineralogy og the Cave no. 4 from Runcului Hill (Metaliferi Mts., Romania). Theoretical and Applied Karstology, 16: 41-46.

Wenrich, K.J., Sutphin, H.B. 1994, Grand Canyon caves, breccia pipes and mineral eposits. Geology Today, 10: 97-104. 